\title{
THE ELECTROCARDIOGRAM IN PECTUS EXCAVATUM
}

\author{
BY \\ JORGE MARTINS DE OLIVEIRA, MOHINDER P. SAMBHI, AND HENRY A. \\ ZIMMERMAN
}

From St. Vincent Charity Hospital, Cleveland, Ohio, U.S.A.

Received December 11, 1957

Pectus excavatum is a deformity of the chest that consists of backward displacement of the sternum and costal cartilages giving rise to a depression in the xiphisternal area. This malformation may appear isolated or associated with other anomalies. The occurrence of pectus excavatum with congenital heart disease has been reported by several observers (Evans, 1946; and McKusick, 1955). Embarrassment of circulation amounting to heart failure has also been described (Ravitch, 1951). Even in the absence of any cardiac condition, electrocardiographic changes are to be expected, because of alterations in the position of the heart. Whether these changes occur as a consequence of cardiac rotation only or also on account of compression of the heart by the chest cage has not yet been established. However, the recent advances in electrocardiography based on vectorial concepts allow us to consider that in cases of pectus excavatum, in the absence of associated cardiac disease, the modifications in the position of the heart are the most important factors, so far as the electrocardiographic changes are concerned.

Previous reports on the electrocardiogram in this malformation exist. Dressler and Roesler (1950) in 10 of their 13 cases, reported inversion and notching of the $T$ waves in præcordial leads (from V1 to V4) as the most remarkable findings. Although most patients in their series were adults, we are not sure that these $T$ wave changes can be considered strictly pathological, since in our experience we have noticed that many normal women retain the juvenile $T$ wave pattern through adult life.

Wachtel et al. (1956), studying 13 other cases, described the changes in QRS complex in lead V1 as the most important finding in pectus excavatum: in eight of these patients, an rsr' pattern was observed. The authors stressed that the above pattern does not indicate disturbance in conduction, but is the consequence of cardiac rotation. They further emphasized the fact that the anatomical configuration of the chest in this malformation, making the correct application of the præcordial lead electrodes difficult, does not permit an exact evaluation of the $T$ wave changes.

\section{ANALYSIS}

Our present study is based on 13 patients with pectus excavatum in whom no evidence of heart disease was found. The ages varied from 5 to 75 years, the average being 24 years. Eight out of these were male and the remaining five were female. Twelve classical leads were recorded in all patients, and in two of them intracavitary electrocardiograms have also been studied. For the sake of convenience, we have divided our analysis into the following three parts. 

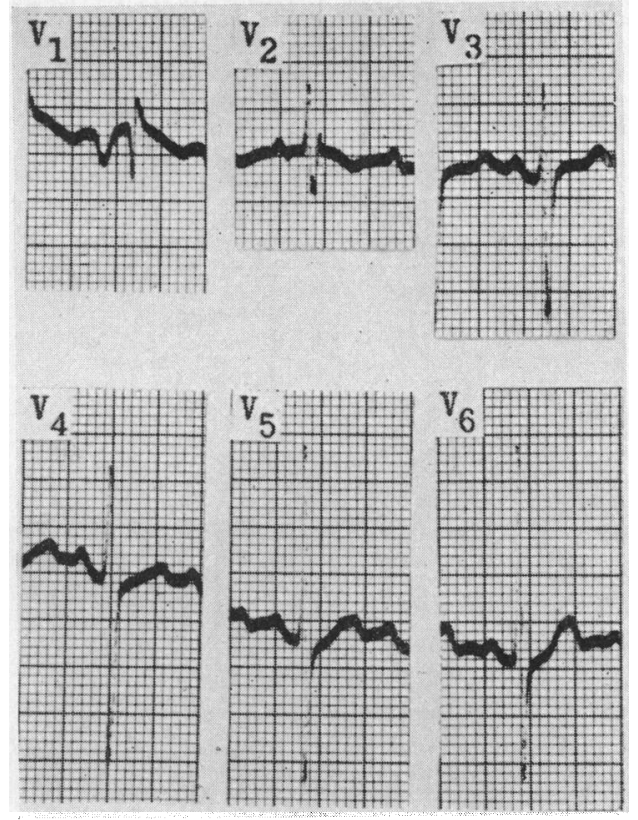

FIG. 1.-Entirely negative $P$ wave in lead V1.

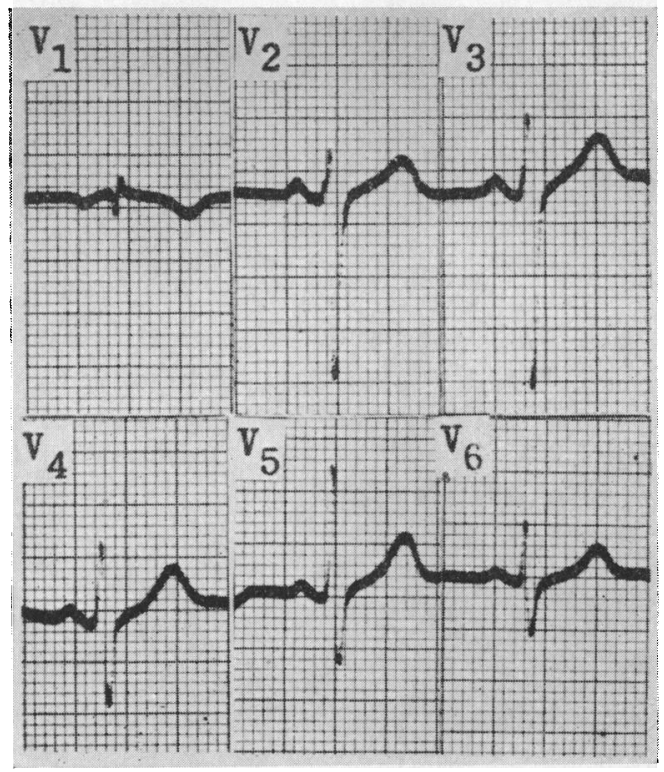

FIG. 2.-qr pattern in V1; disproportional amplitude of the QRS complex in this lead, as compared to V2.

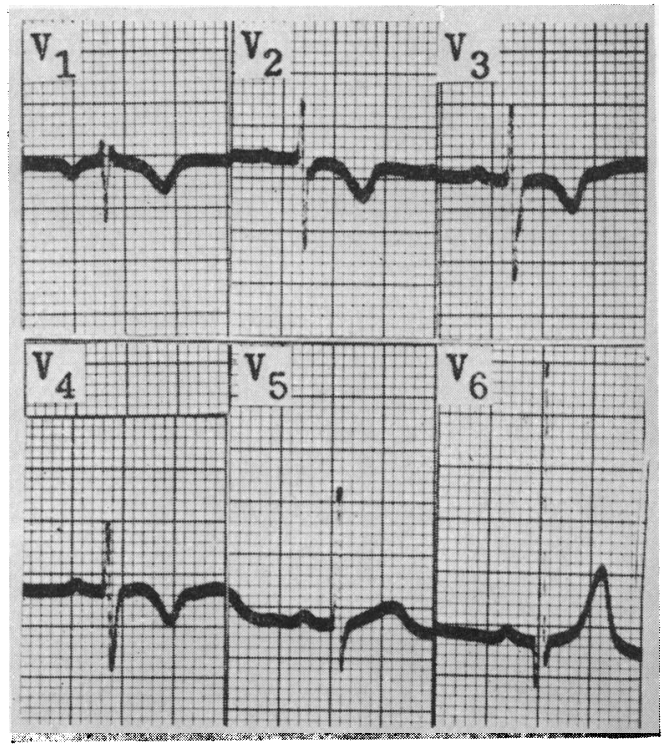

FIG. 3.-rsr' pattern in V1; it was present in 8 out of our 13 cases. 
1. Auricular Activation. The auricular vector was localized between $-25^{\circ}$ and $+75^{\circ}$ in the frontal plane, with a backward orientation in the horizontal plane in all the cases.

$\mathbf{P}$ wave. The duration of this wave in the limb leads was within normal limits in 11 patients; in the remaining two, it exceeded $0 \cdot 10$ seconds. In 11 out of our 13 cases (Fig. 1 ) the $P$ wave was entirely negative in lead V1. In the other two cases, although biphasic, it was predominantly negative. Such an entirely negative $P$ wave in lead V1 is an infrequent finding, even in the presence of marked left auricular hypertrophy or dilatation. In these 11 patients, this pattern of the $P$ wave in the first præcordial lead resembled exactly the one seen in aVR. The reason for this pattern in patients with pectus excavatum will be discussed later.

P-R interval. This was within normal limits in all cases. We did not meet any arrhythmias in our patients with pectus excavatum, as has been reported by other observers (Schaub, and Wegmann, 1954).

2. Ventricular Activation. The duration of the QRS complex was within normal limits in all

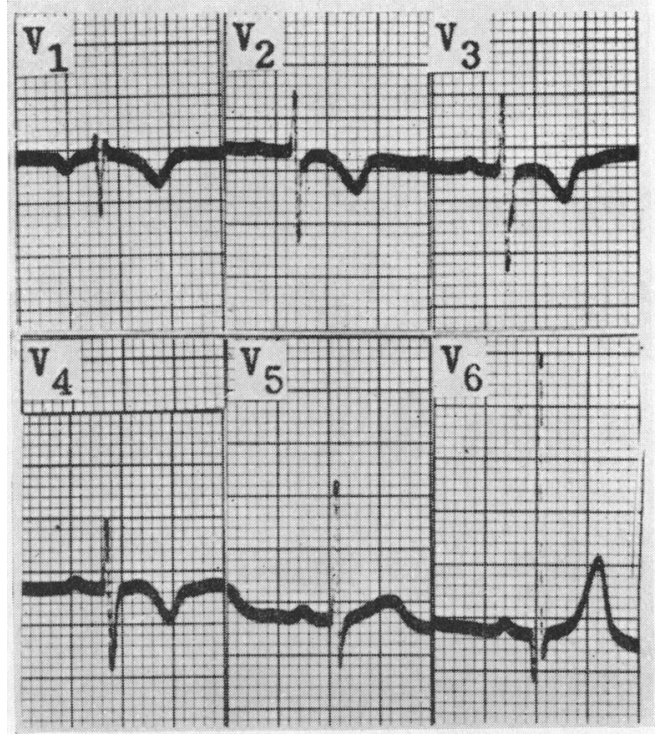

FIG. 4.-Negative $T$ waves from V1 to V4 in a 5-year-old female with pectus excavatum (same case as Fig. 3).

patients. The lead V1 exhibited a qr pattern in four of the cases (Fig. 2) an rsr' pattern in eight (Fig. 3) and a normal $\mathrm{rS}$ pattern in the remaining one.

In lead V2 the pattern of QRS complex was normal in eight patients, but in the other five, the rsr' pattern was present. It seems important to note that the cases which exhibited a $Q$ wave in V1 also presented a disproportionally small total amplitude of the QRS complex in this lead, as compared to V2 (Fig. 2). Such a phenomenon has also been observed frequently in patients with enlarged right auricle. In the limb leads, in all of our cases an $S_{1} S_{3}$ or $S_{1} Q_{3}$ pattern, characterizing a clockwise rotation of the heart, was seen.

SAQRS. It varied between $-100^{\circ}$ and $+110^{\circ}$ in the frontal plane, with a backward orientation in the horizontal plane in all cases. The electrical axis deviated to the right in six patients $(46 \%)$, to the left in one $(8 \%)$, and was within normal limits in the remaining six $(46 \%)$.

3. Ventricular Repolarization. Table I summarizes the pattern of $\mathrm{T}$ waves in the præcordial leads in our patients. 
TABLE I.-Pattern of the $T$ waves in 13 patients with funnel chest.

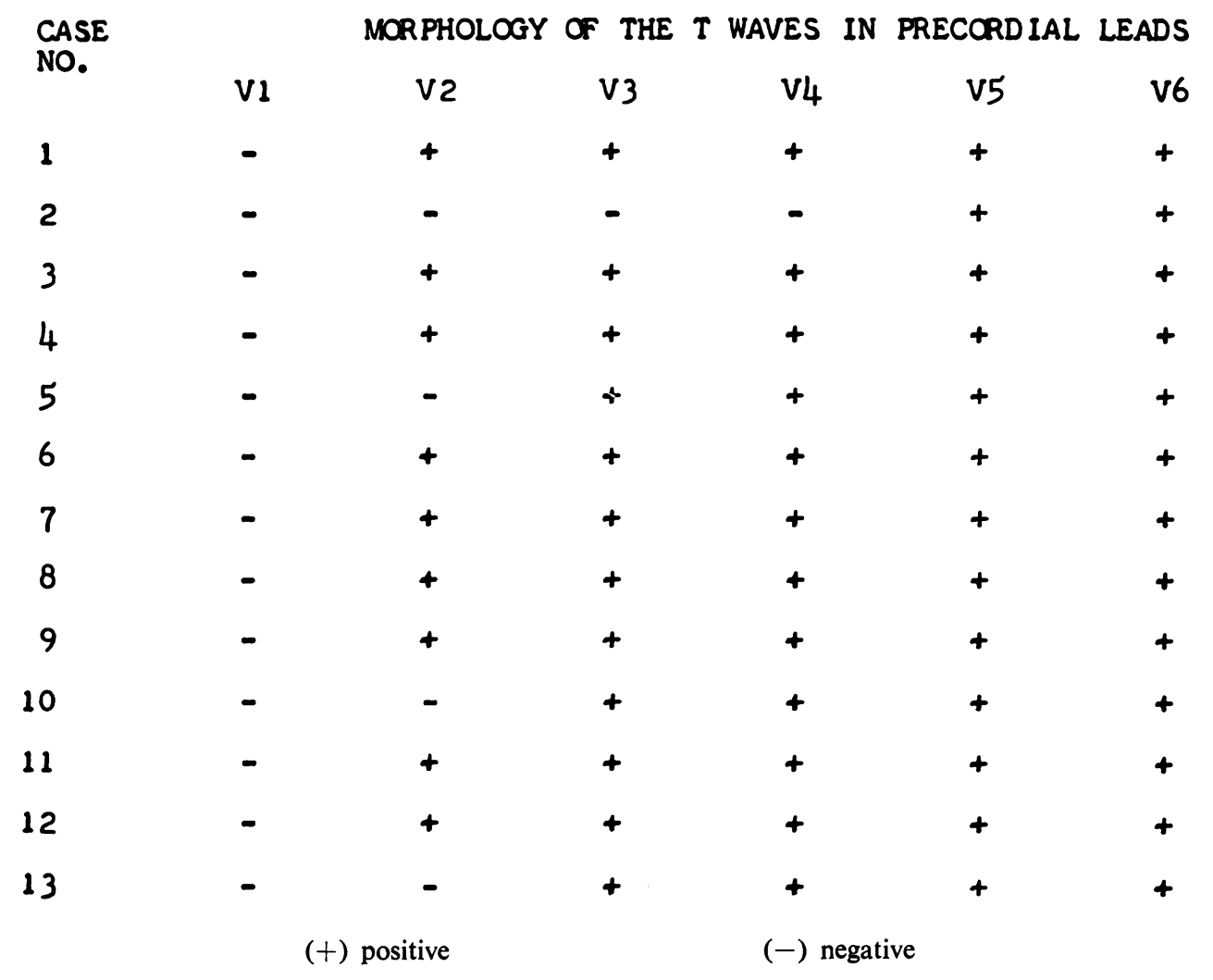

It is evident from Table $\mathrm{I}$ that in all cases the $\mathrm{T}$ wave was negative in lead V1; in four it also appeared negative in V2, and in only one patient did this negativity extend up to V4. We should

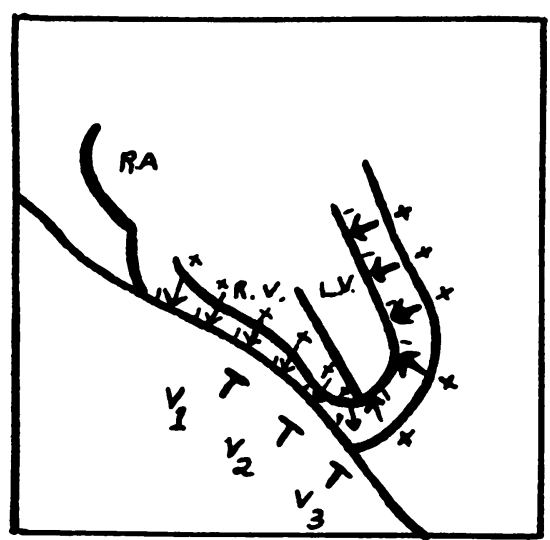

Fig. 5.-Mechanical injury produced by the thoracic deformity causing reversed repolarization. point out, however, that this last patient was a five-year-old girl. Our findings do not agree with those of some other authors (Dressler, and Roesler, 1950) who reported negative $\mathrm{T}$ waves up to $\mathrm{V} 3$ in the majority of their adult patients. As we have emphasized before, we do not think that negative $\mathrm{T}$ waves in right præcordial leads have any special meaning as far as pectus excavatum is concerned. However, in those cases in whom there is compression of the right cardiac chambers by the thoracic deformity, as has been reported by Sweet (1944), an explanation for the negativity of $\mathrm{T}$ waves in right præcordial leads can be suggested. We think that in such cases the mechanical injury thus affecting the epicardium may produce a delay in the initiation of the repolarization in this region. Consequently, the recovery process starts in the subendocardial portions and the orientation of the $T$ vector is reversed (Fig. 5).

Dressler and Roesler (1950) reported a case of pectus excavatum who suffered from typical attacks of angina pectoris and whose electrocardiogram showed negative $T$ waves in multiple præcordial leads. In our own experience, as far as the $\mathrm{T}$ waves in patients with pectus excavatum 
are concerned, the only finding of any statistical significance is the negativity of this wave in lead V1 (all our cases). However, this is not an infrequent finding in normal subjects. The mean vector of ventricular repolarization varied between $0^{\circ}$ and $+65^{\circ}$ in the frontal plane, while a forward orientation in nine cases, and a backward orientation in four was found in the horizontal plane.

\section{Discussion}

Two findings in the electrocardiogram in our cases of pectus excavatum deserve special attention, on account of the constancy with which they appeared: the pattern of the QRS complex in lead V1 and the spatial orientation of the mean auricular activation vector. Wachtel et al. (1956) and Schaub and Wegmann (1954) also have pointed out that in their series, the rsr' pattern was a frequent finding. In a previous communication (Martins de Oliveira and Zimmerman, 1958) we have emphasized that this pattern does not necessarily mean disturbance in conduction in the right branch of the bundle of His itself. It is an established fact (Penaloza and Tranchesi, 1955) that the $r^{\prime}$ wave in the first præcordial lead signifies the depolarization of the basal portion of the right ventricle as well as the higher parts of the interventricular septum, and further, that it is present whenever the mean activation vector of these regions is oriented forward and to the right, either as a consequence of hypertrophy and dilatation of these basal portions or because of a marked rotation of the heart.

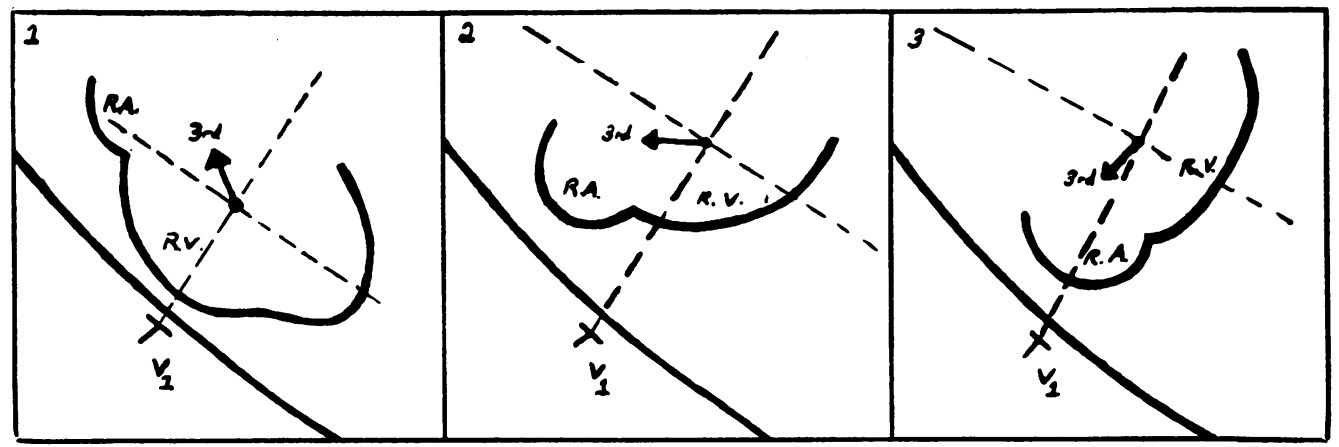

Fig. 6.-Rotation in the position of the heart: (1) Normal position. (2) Backward rotation of the ventricles-the basal (3rd) vector is now oriented to the positive side of V1 which explains the $r^{\prime}$ wave in this lead. (3) Backward rotation of the ventricles and anterior displacement of right auricle-lead V1 now reflects the intracavitary potentials.

The qr pattern in lead V1, as has been stressed by Sodi-Pallares and Calder (1956) represents the transmission of the electrical effects arising from the basal parts of the interventricular septum to the right præcordial leads through an enlarged right auricle. In other words, the right auricular chamber, being situated exactly underneath the exploratory electrode of V1 would permit the direct transmission of intracavitary potentials to that lead.

In cases of pectus excavatum, as a consequence of the thoracic deformity itself, no enlargement would be expected, either of the right auricle or of the basal portions of the right ventricle, as long as no circulatory embarrassment is present. However, a rotation of the heart seems quite evident in these cases. Such a rotation, depending on its degree, may explain the appearance of both rsr' $^{\prime}$ and qr patterns in lead V1 in patients with pectus excavatum. In those cases in 'which there is only a slight rotation, not enough to bring the right auricle to such an anterior position so as to be immediately beneath lead V1, the presence of an $r^{\prime}$ wave may be explained by the rightward and forward deviation of the mean depolarization vector of the basal ventricular portions. On the other hand, cases where the right auricle assumes the position directly below the exploring electrode of $\mathrm{V} 1$, as a consequence of a greater rotation of the heart, this lead now reflects the auricular 
intracavitary potentials and a qr pattern appears (Fig. 6). The simultaneous recording of V1 and of the right auricular cavity obtained in two of our cases, showing the striking similarity of the two tracings, is a strong evidence of this (Fig. 7).

In all our patients, the auricular vector was oriented backwards so producing a negative $P$ wave in lead V1. In spite of the fact that this point has not been considered by other authors (Dressler and Roesler, 1950; Wachtel et al., 1956; Schaub and Wegmann, 1954) the inspection of the records published in their series demonstrates the constant presence of this finding. In our opinion, the

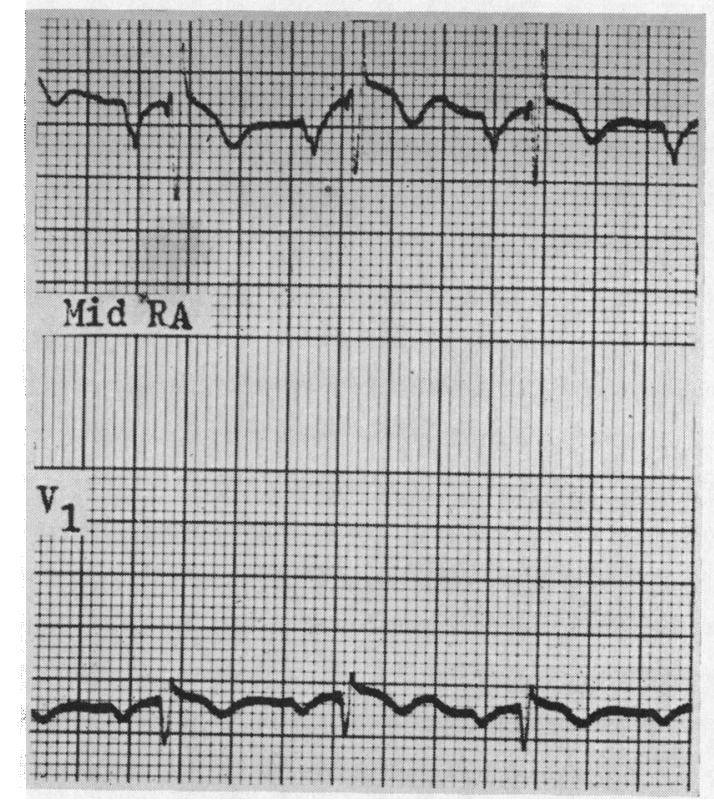

Fig. 7.-Simultaneous recording of $\mathrm{V} 1$ and right auricular cavity in a patient with pectus excavatum. Notice the similarity of the two tracings.

rotation of the heart that had produced these changes in the pattern of the qrs complex seems also to be responsible for this alteration in the orientation of the auricular vector. We further feel that the more anterior situation of the right auricle is the reason for finding $P$ waves in lead V1 very similar to those observed in right auricular intracavitary records.

We are not sure whether the degree of cardiac rotation in cases of pectus excavatum is proportional to the intensity of the thoracic deformity; or if other factors, such as the body build of the patients, are also involved.

Evidently, the electrocardiogram is not an important factor in the primary diagnosis of funnel chest, since this can be established by simple inspection of the thorax. However, as the electrocardiographic changes in this deformity often resemble those seen in certain cardiopathies, as for instance mitral stenosis with pulmonary hypertension (Pruitt and Robinson, 1956) or atrial septal defect (Monroy and Cabrera, 1952), it becomes important to recognize the existence of this chest anomaly in order to avoid a misdiagnosis. Further, the analysis of the electrocardiogram in these cases offers an excellent opportunity to appreciate certain interesting consequences of cardiac rotation.

1. Our observation in two cases faitfully confirmed the experience of other authors (SodiPallares and Calder, 1956; Kossman et al., 1950) regarding the transmission of intracavitary potentials to lead V1, whenever the right atrial chamber is directly below the exploratory electrode. 
2. The presence of a disproportion between the amplitudes of the QRS complex in V1 (small) and V2 (large) is also an argument in favour of the fact that the right auricle is situated immediately below the electrode of V1, since transmitted intracavitary forces obviously will not have the same amplitude as compared to the direct ones from the epicardial surface of the ventricle, as exhibited in $\mathrm{V} 2$ and the rest of the præcordial leads.

3. The frequent appearance of an $\mathrm{rsr}^{\prime}$ pattern in V1 in patients with pectus excavatum gives us a further opportunity to recapitulate an important conclusion that we (Martins de Oliveira and Zimmerman, 1958; and Walker et al., 1956) have stressed before: this pattern does not mean, at least as a rule, a block in the right branch of the bundle of His itself but is the manifestation of vectorial forces transmitted to this præcordial lead, either as an expression of dilatation and hypertrophy of the basal ventricular regions or simply as a result of the rotation of the heart, as it happened in the cases of this present study.

\section{CONCLUSIONS}

The change in cardiac position caused by the thoracic deformity in patients with pectus excavatum produces more or less constant characteristic electrocardiographic findings, which, although not pathognomonic, yet can be suggestive of this anomaly.

These findings consist of: $(a) \mathrm{S}_{1} \mathrm{~S}_{3}$ or $\mathrm{S}_{1} \mathrm{Q}_{3}$; (b) negative $\mathrm{P}$ waves in $\mathrm{V} 1$; and (c) qr or rsr' in VI.

We think these findings are chiefly related to clockwise rotation of the heart on its longitudinal axis, backward orientation of the auricular vector, and transmission of intracavitary potentials to the right præcordial leads.

These electrocardiographic changes, which, for different reasons, can be seen in other conditions like atrial septal defect or mitral stenosis with pulmonary hypertension, may lead to erroneous diagnosis if the electrocardiogram is analysed without previous knowledge of the existence of such thoracic deformity.

The authors gratefully acknowledge the technical assistance of Miss Helen Kleinhenz, R.N., Mrs. Marjorie McIntyre, R.N., and Miss Donna Wolfram.

\section{REFERENCES}

Dressler, W., and Roesler, H. (1950). Amer. Heart J., 40, 877.

Evans, W. (1946). Brit. Heart J., 8, 162.

Kossman, C. E., Berger, A. R., Rader, B., Brumlik, J., Briller, S. A., and Donnelly, J. H. (1950). Circulation, $2,10$. Martins de Oliveira, J., and Zimmerman, H. A. (1958). Amer. Heart J. 55, 369.

Monroy, J. R., and Cabrera, E. (1952). Arch. Inst. Cardiol. Mexico, 22, 330.

McKusick, V. A. (1955). Circulation, 11, 321.

Penaloza, D., and Tranchesi, J. (1955). Amer. Heart J., 49, 51.

Pruitt, R. D., and Robinson, J. G. (1956). Amer. Heart J., 52, 880.

Ravitch, M. M. (1951). Surgery, 30, 178.

Schaub, F., and Wegmann, T. (1954). Cardiologia, 24, 39.

Sodi-Pallares, D., and Calder, R. M. (1956). New Bases of Electrocardiography, 1st English ed. translated from 3rd Spanish ed. The C. V. Mosby Company.

Sweet, R. H. (1944). Ann. Surg., 119, 922.

Wachtel, F. W., Ravitch, M. M., and Grishman, A. (1956). Amer. Heart J., 52, 121.

Walker, J. W., Mattingly, T. W., Pollock, B. E., Carmichael, D. B., Inmon, T. W., and Forrester, R. H. (1956). Amer. Heart J., 52, 547. 